\title{
Bradykinin, insulin, and glycemia responses to exercise performed above and below lactate threshold in individuals with type 2 diabetes
}

\author{
R.Y. Asano ${ }^{1,2}$, R.A.V. Browne ${ }^{3}$, M.M. Sales ${ }^{4}$, G. Arsa ${ }^{5}$, J.F.V.N. Moraes ${ }^{6}$, H.J. Coelho-Júnior ${ }^{7}$, \\ M.R. Moraes ${ }^{8}$, I. Oliveira-Silva ${ }^{9}$, S.E. Atlas ${ }^{10}$, J.E. Lewis ${ }^{11}$ and H.G. Simões ${ }^{8}$ \\ ${ }^{1}$ Curso de Educação Física, Universidade Ibirapuera, São Paulo, SP, Brasil \\ ${ }^{2}$ Curso de Educação Física, Fundação Municipal de Educação Superior de Bragança Paulista, SP, Brasil \\ ${ }^{3}$ Programa de Pós-graduação em Ciências da Saúde, Universidade Federal do Rio Grande do Norte, Natal, RN, Brasil \\ ${ }^{4}$ Departamento de Educação Física, Universidade Estadual de Goiás, Quirinópolis, GO, Brasil \\ ${ }^{5}$ Programa de Pós-graduação Stricto Sensu em Educação Física, Universidade Federal de Mato Grosso, Cuiabá, MT, Brasi \\ ${ }^{6}$ Colegiado de Educação Física, Universidade Federal do Vale do São Francisco, Petrolina, PE, Brasil \\ ${ }^{7}$ Programa de Pós-graduação em Educação Física, Universidade Estadual de Campinas, Campinas, SP, Brasil \\ ${ }^{8}$ Programa de Pós-graduação Stricto Sensu em Educação Física, Universidade Católica de Brasília, Brasília, DF, Brasil \\ ${ }^{9}$ Curso de Educação Física, UniEvangélica, Centro Universitário de Anápolis, Anápolis, GO, Brasil \\ ${ }^{10}$ Department of Medicine, University of Miami Miller School of Medicine, Miami, FL, USA \\ ${ }^{11}$ Department of Psychiatry and Behavioral Sciences, University of Miami Miller School of Medicine, Miami, FL, USA
}

\begin{abstract}
The aim of this study was to analyze the acute responses of bradykinin, insulin, and glycemia to exercise performed above and below lactate threshold (LT) in individuals with type 2 diabetes mellitus (T2D). Eleven participants with a diagnosis of T2D randomly underwent three experimental sessions $72 \mathrm{~h}$ apart: 1) $20 \mathrm{~min}$ of exercise performed at $120 \%$ of LT (120\%LT), 2) 20 min of exercise performed at $80 \%$ of LT $(80 \% \mathrm{LT})$, and 3) $20 \mathrm{~min}$ of control session. Blood glucose was analyzed before, during, and at 45 min post-exercise. Bradykinin and insulin were analyzed before and at $45 \mathrm{~min}$ post-exercise. Both exercise sessions elicited a parallel decrease in glucose level during exercise $(P \leqslant 0.002)$, with a greater decrease being observed for $120 \% \mathrm{LT}(\mathrm{P}=0.005)$. Glucose decreased $22.7 \mathrm{mg} / \mathrm{dL}$ $(95 \% \mathrm{Cl}=10.3$ to $35, \mathrm{P}=0.001)$ at the $45 \mathrm{~min}$ post-exercise recovery period for $80 \% \mathrm{LT}$ and decreased $31.2 \mathrm{mg} / \mathrm{dL}(95 \% \mathrm{Cl}=18.1 \mathrm{to} 44.4$, $P<0.001)$ for $120 \% L T(P=0.004)$. Insulin decreased at post-exercise for $80 \% L T(P=0.001)$ and control $(P \leqslant 0.035)$. Bradykinin increased at 45 min post-exercise only for $80 \% \mathrm{LT}(\mathrm{P}=0.013)$, but was unrelated to the decrease in glucose $(r=-0.16, \mathrm{P}=0.642)$. In conclusion, exercise performed above and below LT reduced glycemia independently of insulin, but exercise above LT was more effective in individuals with T2D. However, these changes were unrelated to the increase in circulating bradykinin.
\end{abstract}

Key words: Metabolic disease; Aerobic exercise; Peptide; Insulin resistance

\section{Introduction}

Blood glucose control is the top priority in the treatment of type 2 diabetes mellitus (T2D), as individuals with T2D and inadequate glycemic control are prone to chronic complications, such as hypertension, retinopathy, nephropathy, dyslipidemia, and other physiological impairments, thereby increasing morbidity and mortality in this population (1). Physical exercise has been extensively recommended for individuals with T2D due to its immediate and long-term effects in lowering blood glucose (2). Furthermore, individuals with T2D should perform aerobic exercise at least at moderate intensity, but higher intensity exercise may be even more effective $(3,4)$.
Exercise can increase blood glucose uptake and its utilization by active skeletal muscle, even with low serum insulin levels. It can serve as an alternative means for glycemic control, since the insulin-dependent pathway is already impaired in T2D (5). However, an exercise-triggered, insulin-independent mechanism for glucose uptake has not yet been fully elucidated. Increasing calcium ions and the activation of pathways responsible for bradykinin release might be associated with blood glucose uptake in an insulin-independent pathway (6).

A classic study conducted by Taguchi et al. (7) demonstrated in rodents and humans the effectiveness of acute 
aerobic exercise in increasing the release and activity of bradykinin in parallel with blood glucose reduction in T2D. Despite the likelihood that exercise-induced bradykinin release would be associated with blood glucose reduction in T2D, to the best of our knowledge no studies have investigated the acute effects of exercise performed above and below lactate threshold on bradykinin, blood glucose, and insulin responses in this population.

We hypothesized that bradykinin release would be exercise intensity-dependent in individuals with T2D, which in turn would influence blood glucose control. In previous studies, we have demonstrated extensively that lactate threshold intensity, which distinguishes domains of moderate to high intensity exercise $(8,9)$, may lead to distinct cardiovascular and metabolic responses during the postexercise recovery period $(10,11)$. Thus, the aim of the present study was to analyze the acute responses of glycemia, insulin and bradykinin to exercise performed above and below lactate threshold in individuals with T2D.

\section{Material and Methods}

\section{Sample}

Subjects participated in a controlled trial with a crossover design. After approval from the Research and Ethics Committee of the Universidade Católica de Brasilia (Protocol No. 167/2011) and signing of the informed consent form (Resolution 466/2012 of the Brazilian National Health Counsel and provisions of the Declaration of Helsinki), 11 individuals (6 women) from the city of Brasilia, clinically diagnosed with T2D participated in the study. Participants were non-smokers with a mean age of $62.1 \pm 9.0$ years. The diagnosis of diabetes was confirmed with a medical evaluation using criteria from the American Diabetes Association (12). All individuals were under medical and nutritional treatment, using one oral hypoglycemic medication (e.g., sulfonylureas, metformin, glibenclamide plus metformin, glimepiride, pioglitazone hydrochloride) and following a diabetic diet to help control their blood glucose.

Exclusion criteria included a diagnosis of peripheral autonomic neuropathy, for which the following aspects were considered: 1) resting heart rate (HR) higher than $90 \mathrm{bpm}$, 2 ) incapable of reaching $85 \%$ of the age-predicted maximum $\mathrm{HR}$ during the maximal incremental exercise test (MIET), 3) a HR reduction less than $12 \mathrm{bpm}$ during the first min after finishing the incremental test, and 4) abnormal HR variability (13). The participants could not have ulcers characteristic of diabetic foot or any other orthopedic impairment that could preclude performing exercise. Furthermore, the individuals could not have been on insulin or any other medicine that could interfere with the outcome variables to be evaluated.

\section{General procedures}

All experimental sessions were performed in the Physical Evaluation and Training Laboratory at the Universidade Católica de Brasilia during the morning,
$2 \mathrm{~h}$ after ingestion of a standard moderate glycemic index $(\mathrm{Gl}=73.9)$ breakfast that provided a total of $315.9 \mathrm{kcal}$, $53 \mathrm{~g}(61.7 \%-212 \mathrm{kcal})$ of carbohydrate, $4.6 \mathrm{~g}(5.8 \%-$ $18.3 \mathrm{kcal})$ of protein, and $9.5 \mathrm{~g}(27.1 \%-85.6 \mathrm{kcal})$ of fat. All medication was washed out for $24 \mathrm{~h}$ prior to the initial screening visit and the three subsequent sessions. The participants were also asked to avoid physical exercise and alcoholic or caffeinated drinks for $24 \mathrm{~h}$ prior to each visit to the laboratory. The participants also underwent a clinical evaluation including a resting electrocardiogram (ErgoPC Elite, Micromed, Brazil), blood pressure (BP) measurements (BP3AC1-1, Microlife Cooperation, Switzerland), anthropometry (body weight, height, and waist circumference), and a maximal incremental exercise test (MIET) on a cycle ergometer (Lode Excalibur Sport, The Netherlands). Body mass index (BMI) was calculated considering the ratio of body weight (2096 PP Standard, Brazil) in kilograms and height (Stadiometer 214, Seca, USA) in squared meters $\left(\mathrm{kg} / \mathrm{m}^{2}\right)$.

\section{Maximal incremental exercise test}

Maximal heart rate $\left(\mathrm{HR}_{\max }\right)$, lactate threshold $(\mathrm{LT})$, and peak oxygen uptake $\left(\mathrm{VO}_{2 \text { peak }}\right)$ were determined during the MIET. After conducting the clinical evaluation, participants performed the MIET on a cycle ergometer with an initial load of $15 \mathrm{~W}$, followed by a $15 \mathrm{~W}$ increase at each 3-min stage at a speed of 60 revolutions per min until volitional exhaustion. During the MIET, a cardiologist monitored the electrocardiogram of the participants. HR, BP, rating of perceived exertion (RPE), ventilation, and blood lactate also were continuously monitored. The following criteria were used to determine whether participants achieved maximal effort: respiratory-exchange ratio (RER) $\geqslant 1.1$, $\mathrm{HR}>90 \%$ age-predicted maximum, and RPE > 17 (14).

Before exercise and during the last $20 \mathrm{~s}$ of each stage, $25 \mu \mathrm{L}$ of capillary blood was drawn from the earlobe, and the samples were collected in Eppendorf microtubes containing $50 \mu \mathrm{L}$ of $1 \%$ sodium fluoride $(\mathrm{NaF})$ for analysis of lactate concentration, using the electro-enzymatic method (Yellow Springs 2.700 STAT, USA). Gas exchange during the MIET was obtained through the facemask of the Metalyzer 3B Gas Analyzer (Cortex Boiphysik, Germany) previously calibrated with a $3-\mathrm{L}$ syringe (calibration flux) and a mixed pattern of gas containing $4.9 \%$ of $\mathrm{CO}_{2}$ and $17 \%$ of $\mathrm{O}_{2}$ (calibration gas). Ventilation, oxygen uptake $\left(\mathrm{VO}_{2}\right)$, and carbon dioxide production $\left(\mathrm{VCO}_{2}\right)$ were registered during the whole procedure, with the last $20 \mathrm{~s}$ of every 3-min stage being analyzed. In addition, BP was measured by the auscultatory method using a sphygmomanometer and a stethoscope (Tycos Hospital Instruments, Brazil) during the last $60 \mathrm{~s}$ of each 3-min stage. All equipment was calibrated according to manufacturers' instructions.

\section{Lactate threshold determination}

To determine the LT, lactate concentration kinetics were examined during the MIET stages according to Simões 
et al. (9). Based on this previous report, LT was identified in individuals with T2D as an exercise intensity (Watts) above which the blood lactate concentration increases overproportionally in relation to the workload increase. It is important to point out that LT intensity is similar to both blood glucose threshold and ventilatory thresholds (9).

\section{Experimental sessions ( 80 and $120 \%$ of LT intensity)}

In these sessions, the volunteers performed 20 min of aerobic exercise on a cycle ergometer (Lode Excalibur Sport) with a relative intensity of $80 \%(80 \% \mathrm{LT}$ : moderate intensity) and $120 \%$ (120\%LT: high intensity) of the LT workload that was previously determined during the MIET. A fixed 20-min exercise was chosen to avoid effects of exercise duration on endocrine and perceptual physiological responses that are time-dependent, as previously described $(15,16)$. The order of sessions [80\%LT, 120\%LT, and control $(\mathrm{CON})]$ was randomized, and the minimum and maximum intervals between sessions were 72 and $120 \mathrm{~h}$, respectively. To try to isolate the effect of exercise on metabolic activity, individuals were prevented from receiving any visual stimulus in the recovery period. Therefore, no activity, such as reading, was allowed during this period. Thus, individuals were instructed to remain seated until the 45th min.

\section{Control session}

The control followed the same procedures applied in $80 \%$ LT and $120 \%$ LT. However, the participants remained seated in a resting position without performing exercise.

\section{Measurements performed in the experimental sessions}

In all sessions, at 15 min of pre-exercise rest, a $25 \mu \mathrm{L}$ blood sample was collected from the earlobe (glucose). Blood samples were taken every 5 min during the $20 \mathrm{~min}$ of either exercise $(80 \%$ LT or $120 \% \mathrm{LT})$ or CON, as well as at 15 and 45 min of the post-session recovery period. Capillary blood samples were collected and analyzed similarly to blood lactate measures.

Furthermore, at 15-min of pre-exercise rest and at the 15th and 45th min of post-intervention recovery, a blood sample $(8 \mathrm{~mL})$ was collected from the antecubital or radial vein (bradykinin and insulin) and was deposited in two 4-mL tubes (Vacuette, BD, USA). Samples were centrifuged at room temperature for $15 \mathrm{~min}$ at $1509.3 \mathrm{~g}$ Plasma aliquots were separated in Eppendorf microtubes containing inhibitors of kinin degradation - orthophenanthroline, ethylenediamine tetraacetic acid (EDTA), dipyridyl, and sodium tetrathionate - and immediately stored at $-20^{\circ} \mathrm{C}$.

$\mathrm{VO}_{2}, \mathrm{HR}$ and lactate were monitored every $5 \mathrm{~min}$ (5th, 10th, 15th and 20th $\mathrm{min}$ ) during the sessions and the average was calculated.

\section{Insulin}

To measure serum insulin, we used human insulin enzyme-linked immunosorbent assay (ELISA) kit plate
(Millipore). First, the wash buffer was diluted 10-fold by $450 \mathrm{~mL}$ of distilled water. After that, each well of the 96well plate was filled with $300 \mu \mathrm{L}$ of wash buffer and incubated for $5 \mathrm{~min}$ at room temperature $\left(28^{\circ} \mathrm{C}\right)$. The assay buffer was then added to all control and sample wells except those that were used to generate the standard curve. Procedures were performed according to kit instructions.

\section{Bradykinin}

Plasma bradykinin extraction was carried out using SepPak C18 (Waters Corp., USA) columns previously activated with $90 \%$ acetonitrile $(2 \mathrm{~mL})$, water $(5 \mathrm{~mL})$, and $5 \%$ acetonitrile in $1 \%$ phosphoric acid $(5 \mathrm{~mL})$. Subsequent to the activation, the samples were applied in the column, washed with $5 \%$ acetonitrile in $1 \%$ phosphoric acid, and eluted at $35 \%$ acetonitrile in $1 \%$ phosphoric acid. The eluates were lyophilized, dissolved again in $500 \mu \mathrm{L}$ of mobile phase $\mathrm{A}$ ( $5 \%$ acetonitrile in $0.1 \%$ orthophosphoric acid), and filtered through a $0.22 \mu \mathrm{m}$ membrane for analysis by high performance liquid chromatography. The peptides were then separated on a reversed phase column (Aquapore 300 ODS; $250 \times 4.6 \mathrm{~mm}$ ), using isocratic gradient for $5 \mathrm{~min}$ followed by 20 min of linear gradient from $5 \%$ to $35 \%$ mobile phase $\mathrm{B}\left(95 \%\right.$ acetonitrile in $\left.0.1 \% \mathrm{H}_{3} \mathrm{PO}_{4}\right)$, under a flow of $1.5 \mathrm{~mL} / \mathrm{min}$ for a period of $40 \mathrm{~min}$. Bradykinin was identified by comparing the retention time with the standard kinin. All samples were tested in duplicate and the average values were considered.

\section{Statistical analysis}

Data normality was tested using Shapiro-Wilk's test; skewness and kurtosis (z-score) were also tested. Descriptive variables are reported as mean $\pm S D$ for parametric data and median and interquartile range (25th-75th percentile) for nonparametric data. Repeated measures ANOVA followed by Bonferroni's post hoc test was used to compare the values of $\mathrm{VO}_{2}$, blood lactate, $\% \mathrm{HR}_{\max }$, work rate, and area under the curve (AUC) of blood glucose, insulin and bradykinin levels between conditions. Friedman's ANOVA followed by pairwise comparison with Bonferroni's correction was used to verify the effect of time and condition on the blood glucose level during experimental conditions. The effect size of the post hoc test for nonparametric data was calculated by the formula $r=z / \sqrt{ } N$.

A two-way (condition vs time) repeated measures ANOVA was used to analyze blood glucose, insulin, and bradykinin levels following the experimental conditions. Partial eta squared (partial $\eta^{2}$ ) was used to determine the effect size of the variance. In the case of a sphericity assumption violation, the degrees of freedom were adjusted and reported using the Greenhouse-Geisser epsilon correction. When necessary, Bonferroni's post hoc test was used to find significant differences. Spearman's correlation coefficient was used to examine the relationship between the absolute change in blood glucose and the absolute 
change in bradykinin in each experimental condition. The level of statistical significance was $\mathrm{P}<0.05$. All data were analyzed using SPSS version 22.0 for Windows (IBM, Inc., USA).

The clinical significance was evaluated using an approach based on the magnitudes of change (17). Cohen's $\mathrm{d}$ was used to determine the effect size of absolute change between control and experimental sessions. The effect size magnitude was based in the Hopkins's scale: $<0.2$ is trivial, $0.2-0.5$ is small, $0.6-1.1$ is moderate, $1.2-1.9$ is large and 2.0 or more is very large (18). For betweensession comparisons, the chance that the true (unknown) values for each session were beneficial, unclear or harmful for change was calculated. Quantitative chances of beneficial or harmful effect were assessed qualitatively as follows: $<1 \%$, almost certainly not; $1-5 \%$, very unlikely; 5-25\%, unlikely; 25-75\%, possibly; 75-95\%, likely; 95-99\%, very likely; and $>99 \%$, almost certainly. If the chances of having beneficial or harmful changes were both $>10 \%$, the true difference was considered unclear (17)

A post hoc statistical power analysis for the differences in the glucose responses between the three experimental conditions (i.e., pre- vs post-exercise) was conducted to determine the achieved power, based on the sample size $(n=11)$, an alpha of 0.05 , and the achieved effect size. For two-way repeated measures ANOVA, the achieved power for the interaction group by time was $92 \%$ and the main effect of time was $100 \%$.

\section{Results}

The main characteristics of the participants were $62.1 \pm 9.0$ years old, body weight of $74.8 \pm 12.2 \mathrm{~kg}$, height of $1.61 \pm 0.1 \mathrm{~m}$, BMl of $28.8 \pm 4.6 \mathrm{~kg} / \mathrm{m}^{2}$, waist circumference of $90.3 \pm 10.0 \mathrm{~cm}$, fasting glucose of $154.7 \pm$ $56.8 \mathrm{mg} / \mathrm{dL}, \mathrm{VO}_{2}$ peak of $21.4 \pm 4.5 \mathrm{~mL} \cdot \mathrm{kg}^{-1} \cdot \mathrm{min}^{-1}$, systolic blood pressure of $129.5 \pm 10.1 \mathrm{mmHg}$, and diastolic blood pressure of $73.1 \pm 10.3 \mathrm{mmHg}$. The mean fasting glucose value demonstrated a hyperglycemic condition, and the $\mathrm{VO}_{2}$ peak revealed low physical fitness.
Table 1 shows the results of $\mathrm{VO}_{2}$, lactate, $\% \mathrm{HR}_{\max }$, exercise time and total work during the different experimental sessions ( $80 \% \mathrm{LT}, 120 \% \mathrm{LT}$ and CON), evidencing the differences of exercise intensities on studied parameters. Because a fixed 20-min exercise was chosen, not only the intensity but also total work was different between sessions. The mean results from all time-points of the 20-min experimental sessions revealed that $\mathrm{VO}_{2}$ was significantly higher in $120 \% \mathrm{LT}$ compared to $80 \% \mathrm{LT}$ and CON, and in $80 \% \mathrm{LT}$ compared to CON $(\mathrm{P}<0.05)$. In addition, the \% of maximal HR was different between exercise sessions $(P<0.001)$. Furthermore, the final blood lactate measurement was higher only in $120 \%$ LT compared to CON, evidencing the higher exercise intensity (internal work) for the $120 \% \mathrm{LT}$.

Figure 1 shows the blood glucose levels during experimental conditions. Regarding 120\%LT, the effect of time was significant $\left(\chi^{2}(4)=31.6, \mathrm{P}<0.001\right)$. Post hoc pairwise

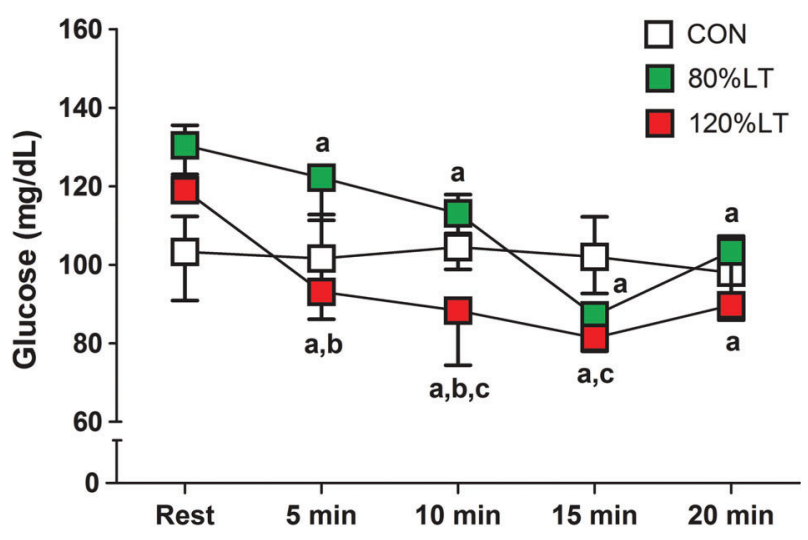

Figure 1. Blood glucose at each 5-min stage during the control, $80 \% \mathrm{LT}$, and $120 \% \mathrm{LT}$ sessions. Data are reported as median, 25th and 75th percentiles). CON: control condition; $80 \% \mathrm{LT}$ : exercise at $80 \%$ of lactate threshold; $120 \% \mathrm{LT}$ : exercise at $120 \%$ of lactate threshold. ${ }^{\text {ap }}<0.0125$ compared to rest at the same session; ${ }^{\mathrm{b}} \mathrm{P}<0.0167$ compared to $80 \% \mathrm{LT}$ at the same time; ${ }^{\mathrm{C}} \mathrm{P}<0.0167$ compared to CON at the same time (Friedman's ANOVA).

Table 1. Mean values for $\mathrm{VO}_{2}$, blood lactate, $\%$ maximal $\mathrm{HR}$ and total work during the exercise $(80 \% \mathrm{LT}$ and $120 \% \mathrm{LT})$ and control conditions.

\begin{tabular}{lccc}
\hline & CON & $80 \% \mathrm{LT}$ & $120 \% \mathrm{LT}$ \\
\hline Time $(\mathrm{min})$ & 20 & 20 & 20 \\
$\mathrm{VO}_{2}\left(\mathrm{~mL} \cdot \mathrm{kg}^{-1} \cdot \mathrm{min}^{-1}\right)$ & $2.9 \pm 0.4$ & $12.9 \pm 2.6^{\mathrm{a}}$ & $17.6 \pm 3.0^{\mathrm{a}, \mathrm{b}}$ \\
Lactate $^{\mathrm{m} m \mathrm{~mm} / \mathrm{L})}$ & $1.3 \pm 0.5$ & $2.8 \pm 0.9$ & $5.2 \pm 1.5^{\mathrm{a}}$ \\
$\mathrm{HR}_{\max }(\%)$ & $46.5 \pm 6.9$ & $77.5 \pm 7.1$ & $92.6 \pm 11.1$ \\
Work $(\mathrm{kJ})$ & $93.1 \pm 20.8$ & $352.1 \pm 91.8^{\mathrm{a}}$ & $557.1 \pm 152.3^{\mathrm{a}, \mathrm{b}}$ \\
\hline
\end{tabular}

CON: control session; $80 \% \mathrm{LT}$ : exercise condition at $80 \%$ of lactate threshold; $120 \% \mathrm{LT}$ : exercise condition at $120 \%$ of lactate threshold; $\mathrm{VO}_{2}$ : oxygen consumption; $\mathrm{HR}_{\max }$ : maximal heart rate. ${ }^{a} \mathrm{P}<0.05$ compared to $\mathrm{CON}$; ${ }^{\mathrm{b}} \mathrm{P}<0.05$ compared to $80 \% \mathrm{LT}$ (repeated measures ANOVA). 
comparisons reveled that glucose decreased at the 5th $(P=0.002, r=0.86)$, 10th $(P=0.001, r=0.88)$, 15th $(P=0.001$, $r=0.88)$, and 20th $(P=0.001, r=0.89)$ min during exercise. Also, the effect of time was significant for $80 \% \mathrm{LT}\left(\chi^{2}(4)=\right.$ 38.5, $\mathrm{P}<0.001)$, with post hoc pairwise comparisons revealing that glucose decreased at the 5th $(P=0.001$, $r=0.89)$, 10th $(P=0.001, r=0.88)$, 15th $(P=0.001, r=0.88)$, and 20th $(P=0.001, r=0.88)$ min during exercise. Regarding CON, the effect of time was significant $\left(\chi^{2}(4)=9.7\right.$, $\mathrm{P}=0.04$ ), but post hoc pairwise comparisons reveled no significant changes in glucose levels between rest and during exercise.

Regarding the effect of condition, the 5th min during exercise was significant $\left(\chi^{2}(2)=6.7, \mathrm{P}=0.04\right)$. Post hoc pairwise comparisons revealed that the decrease in glucose was higher in $120 \% \mathrm{LT}$ compared to $80 \% \mathrm{LT}$ ( $r=$ $-0.80, \mathrm{P}=0.005)$. For the 10th min, the effect was significant $\left(\chi^{2}(2)=8.9, P=0.01\right)$ since post hoc pairwise comparisons revealed that the decrease in glucose was also higher in $120 \% \mathrm{LT}$ compared to $80 \% \mathrm{LT}(\mathrm{P}=0.005, \mathrm{r}=-0.80)$ and CON $(P=0.007, r=-0.78)$. In the 15th min, the effect was also significant $\left(\chi^{2}(2)=12.2, P=0.001\right)$, when the post hoc pairwise comparisons revealed that glucose decreased in $120 \%$ LT more than in CON ( $P=0.003, r=-0.83)$. Regarding the 20th $\mathrm{min}$, the effect was marginally insignificant $\left(\chi^{2}(2)=5.6, P=0.06\right)$.

Figure 2 shows the blood glucose, insulin, and bradykinin levels at the 15th and 45th min of post-exercise recovery during the experimental sessions. For glucose, the main effect of time was significant $(F(2,20)=35.8, P<0.001$, partial $\left.\eta^{2}=0.78\right)$, and the condition $\times$ time interaction was also significant $\left(F(2.6,26.1)=6.3, P=0.003\right.$, partial $\eta^{2}=$ 0.39). The effect of condition was non-significant $(F(1.3$, $12.9)=0.98, P=0.36$, partial $\eta^{2}=0.09$ ). Bonferroni's post hoc analysis revealed that glucose decreased at the 15th and 45th min of post-exercise recovery in $80 \% \mathrm{LT}$ and $120 \%$ LT compared to rest in the same session $(P<0.001)$. Additionally, the $120 \% \mathrm{LT}$ session decreased more than the $80 \%$ LT after 45 min post-exercise $(P=0.004)$.

Regarding insulin, the main effects of time $(F(1.3$, 12.8) $=36.9, P<0.001$, partial $\left.\eta^{2}=0.79\right)$ and condition $(F(2$, $20)=7.8, P=0.003$, partial $\eta^{2}=0.44$ ) were significant. The condition $\times$ time interaction was also insignificant $(F(2.2$, 21.8)=1.6, $P=0.223$, partial $\left.\eta^{2}=0.14\right)$. Bonferroni's post hoc analysis revealed that insulin decreased at the 15th and 45th min of post-exercise recovery in $80 \% \mathrm{LT}(\mathrm{P}=$ $0.001)$ and $C O N(P \leqslant 0.04)$ compared to rest in the same session. Furthermore, the $80 \% \mathrm{LT}$ session decreased more than CON at the 15th min of post-exercise recovery $(P=$ 0.03 ), and insulin decreased more at the 45th min in $80 \% \mathrm{LT}$ $(P=0.002)$ and $120 \% \mathrm{LT}(\mathrm{P}=0.004)$ compared to CON.

For bradykinin, the main effect of time $(F(2,20)=$ 15.4, $\mathrm{P}<0.001$, partial $\eta^{2}=0.61$ ) and the condition $\times$ time interaction $\left(F(4,40)=4.5, P=0.005\right.$, partial $\left.\eta^{2}=0.31\right)$ were significant. Bonferroni's post hoc analysis revealed that bradykinin decreased at the 15th min of post-exercise


Figure 2. Blood glucose $(A)$, insulin $(B)$, and bradykinin $(C)$ levels after the control (CON), $80 \% \mathrm{LT}$, and $120 \% \mathrm{LT}$ sessions. Data are reported as means \pm SD. CON: control condition; $80 \% \mathrm{LT}$ : exercise at $80 \%$ of lactate threshold; $120 \%$ LT: exercise at $120 \%$ of lactate threshold; Rec: recovery. ${ }^{\mathrm{a}} \mathrm{P}<0.05$ compared to rest in the same session; ${ }^{b} \mathrm{P}<0.05$ compared to $\mathrm{CON}$ at the same time point; ${ }^{\mathrm{C}} \mathrm{P}<0.05$ compared to $80 \% \mathrm{LT}$ at the same time point of the session time (two-way ANOVA).

recovery in $120 \% \mathrm{LT} \quad(\mathrm{P}<0.001)$ and CON $(\mathrm{P}=0.006)$ compared to rest in the same session. Also, bradykinin increased at the 45th min of post-exercise recovery only in $80 \% \mathrm{LT}(\mathrm{P}=0.013)$ compared to rest in the same session. In addition, the effect of condition was significant 
$\left(\mathrm{F}(2,20)=6.7, \mathrm{P}=0.006\right.$, partial $\left.\eta^{2}=0.40\right)$. Bonferroni's post hoc analysis revealed that the bradykinin baseline level was different between CON and $80 \% \mathrm{LT}(\mathrm{P}=0.001)$ and between $80 \%$ LT and $120 \%$ LT $(P<0.001)$.

Table 2 shows the clinical significance based in the magnitude of absolute change between control and experimental sessions. Regarding the main outcome (i.e., postexercise blood glucose level), the $80 \% \mathrm{LT}$ demonstrated a moderate effect size and was very likely of being clinically beneficial at both post-exercise time points. Furthermore, the $120 \%$ LT showed a moderate effect size and was very likely clinically beneficial at the first postexercise time point and almost certainly clinically beneficial at the last post-exercise time point (i.e., $45 \mathrm{~min}$ post-exercise).

Figure 3 shows the total AUC between baseline and the 15th and 45th min of post-exercise recovery of glucose, insulin, and bradykinin levels following the experimental sessions. There was no effect of condition for glucose $\left(F(1.3,12.6)=1.52, P=0.248\right.$, partial $\left.\eta^{2}=0.13\right)$ and bradykinin $\left(F(2,20)=2.07, P=0.152\right.$, partial $\left.\eta^{2}=0.17\right)$. Regarding insulin, there was an effect of condition $(F(2$, $20)=8.94, P=0.002$, partial $\eta^{2}=0.47$ ). Bonferroni's post hoc analysis revealed that insulin decreased in $80 \% \mathrm{LT}$ $(P=0.024)$ and $120 \% L T(P=0.021)$ compared to the CON.
Figure 4 shows the Spearman's correlation coefficient between delta values [rec $45 \mathrm{~min}$ - rest] of blood glucose and of bradykinin in the $80 \%$ LT and $120 \%$ LT conditions. The change in glucose was not related to the change in bradykinin in the $80 \% \mathrm{LT}$ condition ( $\mathrm{r}=-0.16, \mathrm{P}=0.64)$. Regarding $120 \% \mathrm{LT}$, the change in glucose was significantly correlated with the change in bradykinin postexercise $(r=0.64, P=0.03)$.

\section{Discussion}

The main finding of the present study was the effectiveness of aerobic exercise prescription based on LT intensity for individuals with T2D. The glycemia of participants decreased during and after exercise performed above and below the LT in T2D patients. However, the exercise session performed at $120 \% \mathrm{LT}$ was more effective than $80 \% \mathrm{LT}$ to decrease glucose during and after exercise, with no effect on total AUC for glycemia. The decrease in glycemia occurred with low insulin, suggesting a non-insulin dependent pathway of glucose uptake after exercise. In turn, bradykinin increased only $45 \mathrm{~min}$ after exercise performed at $80 \% \mathrm{LT}$, with no effects of exercise sessions on total AUC, and this was unrelated to the decrease in glucose. Thus, the null hypothesis of this

Table 2. Clinical significance following the exercise conditions.

\begin{tabular}{|c|c|c|c|c|c|c|}
\hline & \multirow[t]{2}{*}{ ES and $90 \% \mathrm{CL}$} & \multirow[t]{2}{*}{ Magnitude } & \multicolumn{3}{|c|}{ Quantitative chance (\%) } & \multirow[t]{2}{*}{ Qualitative chance } \\
\hline & & & Harmful & Trivial & Beneficial & \\
\hline \multicolumn{7}{|l|}{ Glucose } \\
\hline \multicolumn{7}{|l|}{ 15-min post } \\
\hline $80 \% \mathrm{LT}$ & $-0.62(-0.95$ to -0.30$)$ & Moderate & $0 \%$ & $2 \%$ & $98 \%$ & Very likely \\
\hline $120 \% \mathrm{LT}$ & $-0.85(-1.36$ to -0.33$)$ & Moderate & $0 \%$ & $2 \%$ & $98 \%$ & Very likely \\
\hline \multicolumn{7}{|l|}{ 45-min post } \\
\hline $80 \% \mathrm{LT}$ & $-0.60(-0.98$ to -0.22$)$ & Moderate & $0 \%$ & $4 \%$ & $96 \%$ & Very likely \\
\hline $120 \%$ LT & $-0.94(-1.41$ to -0.47$)$ & Moderate & $0 \%$ & $1 \%$ & $99 \%$ & Almost certainly \\
\hline \multicolumn{7}{|l|}{ Insulin } \\
\hline \multicolumn{7}{|l|}{ 15-min post } \\
\hline $80 \% \mathrm{LT}$ & $-0.21(-0.62$ to 0.20$)$ & Small & $5 \%$ & $44 \%$ & $51 \%$ & Possibly \\
\hline $120 \%$ LT & $0.43(-0.19$ to 1.04$)$ & Small & $74 \%$ & $21 \%$ & $5 \%$ & Possibly \\
\hline \multicolumn{7}{|l|}{ 45-min post } \\
\hline $80 \% \mathrm{LT}$ & $-0.46(-0.90$ to -0.01$)$ & Small & $1 \%$ & $15 \%$ & $84 \%$ & Likely \\
\hline $120 \%$ LT & $0.06(-0.61$ to 0.74$)$ & Trivial & $36 \%$ & $39 \%$ & $25 \%$ & Unclear \\
\hline \multicolumn{7}{|l|}{ Bradykinin } \\
\hline \multicolumn{7}{|l|}{ 15-min post } \\
\hline $80 \% \mathrm{LT}$ & 1.84 (0.94 to 2.75$)$ & Large & $0 \%$ & $0 \%$ & $100 \%$ & Almost certainly \\
\hline $120 \% \mathrm{LT}$ & $-0.25(-1.23$ to 0.74$)$ & Small & $53 \%$ & $25 \%$ & $21 \%$ & Unclear \\
\hline \multicolumn{7}{|l|}{ 45-min post } \\
\hline $80 \%$ LT & $1.15(0.16$ to 2.13$)$ & Moderate & $2 \%$ & $4 \%$ & $94 \%$ & Likely \\
\hline $120 \% \mathrm{LT}$ & $-0.72(-2.32$ to 0.88$)$ & Moderate & $71 \%$ & $12 \%$ & $16 \%$ & Unclear \\
\hline
\end{tabular}

Effect size (ES) are Cohen's d (absolute change in control vs absolute change in exercise) and 90\% confidence limits (90\% CL). 80\%LT: exercise at $80 \%$ of lactate threshold; $120 \%$ LT: exercise at $120 \%$ of lactate threshold. 

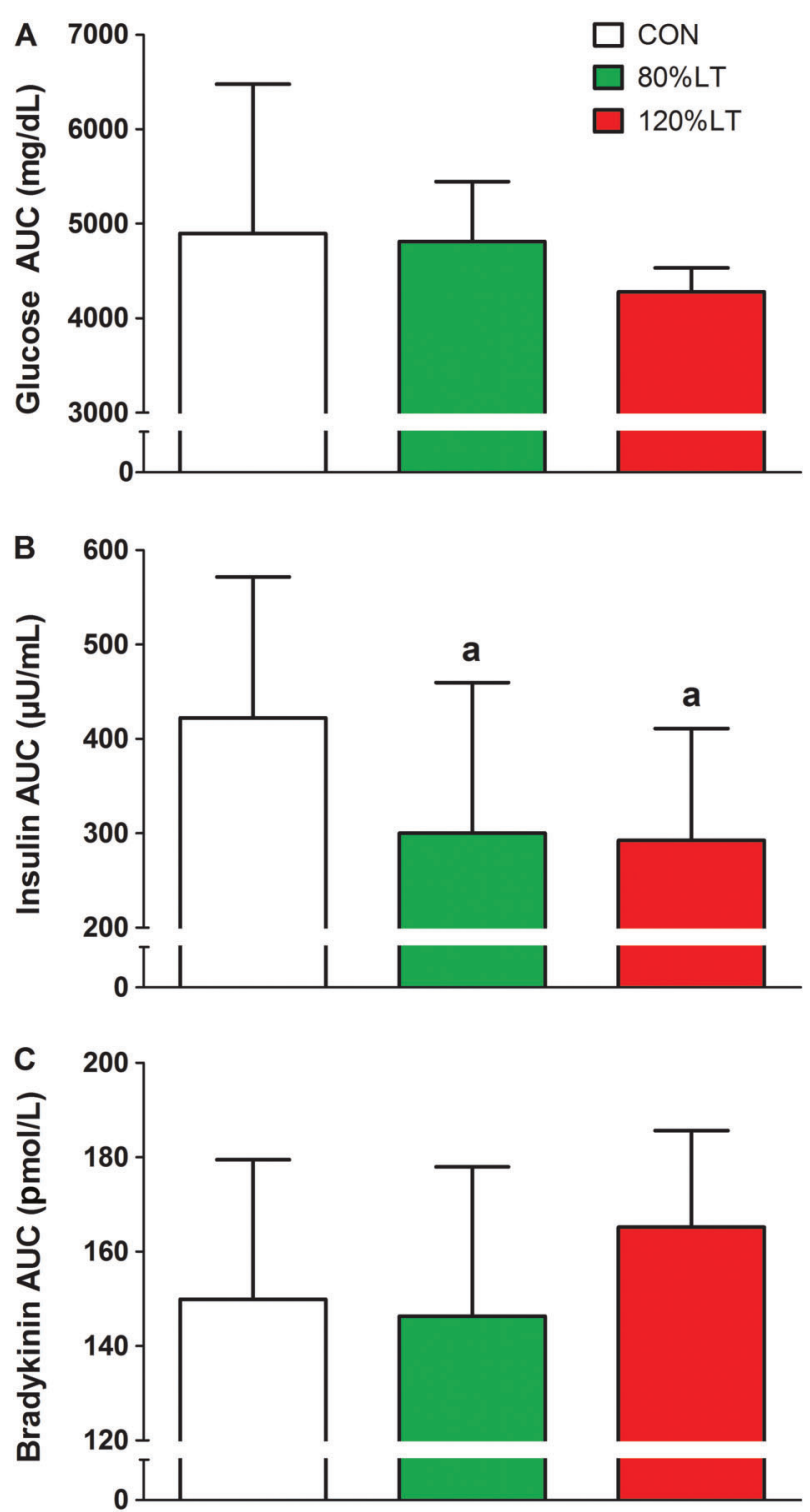

Figure 3. Area under the curve (AUC) of blood glucose $(A)$, insulin $(B)$, and bradykinin $(C)$ after the CON, $80 \% \mathrm{LT}$, and $120 \%$ LT sessions. Data are reported as means \pm SD. CON: control condition; $80 \% \mathrm{LT}$ : exercise at $80 \%$ of lactate threshold; $120 \% \mathrm{LT}$ : exercise at $120 \%$ of lactate threshold. ${ }^{\text {ap }}<0.05$ compared to CON (repeated measures ANOVA).

study was supported, i.e., glucose uptake occurred without changes in plasma bradykinin in individuals with T2D.

The American College of Sports Medicine and the American Diabetes Association guidelines suggest that individuals with $\mathrm{T} 2 \mathrm{D}$ perform aerobic exercise at moderate intensity $\left(40-60 \% \mathrm{VO}_{2 \max }\right)$ (19). However, exercise at high intensity is encouraged, since it confers additional benefits $(3,4)$. Meta-analytic data showed a higher potential for long-term high intensity exercise to increase



$\Delta$ Bradykinin (pmol/L)

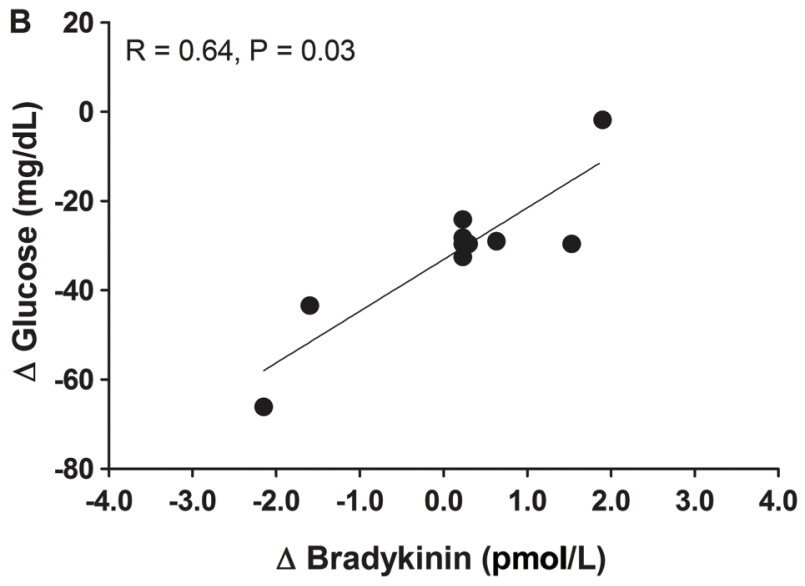

Figure 4. Spearman's correlation coefficient between absolute change [rec $45 \mathrm{~min}$ - rest] of blood glucose and bradykinin in the conditions of exercise at $80 \%$ of lactate threshold $(A)$ and at $120 \%$ of lactate threshold $(B)$.

$\mathrm{VO}_{2 \max }$ and decrease glycated hemoglobin compared to moderate exercise (3). Moreover, these alterations were not associated with the modulation of other exercise parameters, such as volume and frequency of exercise (3). However, no consensus exists about which exercise intensity can cause greater improvements in physiological systems (mainly on glucose level regulation) in individuals with T2D $(3,19)$.

In the present study, exercise sessions performed in different exercise-intensity domains (i.e., above and below $\mathrm{LT}$, eliciting distinct physiological responses as observed in Table 1) were selected based on their possible effects on glycemia. In previous studies from our research group, in which exercise intensities below and slightly above the LT (i.e., $10 \%$ below and $10 \%$ above LT) were analyzed $(11,20)$, a blood glucose reduction was observed as exercise intensity increased. However, in the present investigation a higher intensity $(20 \%$ above LT) was tested with wash-out medications. 
The volunteers showed a greater decrease in blood glucose during and after exercise performed at $120 \% \mathrm{LT}$ compared to $80 \% \mathrm{LT}$, and the clinical relevance was very likely to both intensities after 15 min post-exercise, but seemed to be more effective for $120 \%$ LT after 45 min of post-exercise recovery. However, in a previous study when ten individuals with T2D $(56.9 \pm 11.2$ years, $80.3 \pm 14.4 \mathrm{~kg}$, $1.68 \pm 0.09 \mathrm{~m}$, and $\mathrm{VO}_{2 \max }$ of $18.0 \pm 3.6 \mathrm{~mL} \cdot \mathrm{kg}^{-1} \cdot \mathrm{min}^{-1}$ ) performed two sessions of exercise above and below LT, both sessions elicited comparable decreases in blood glucose (11). Similarly, Gross (21) did not observe any difference on glucose uptake among five young adults performing high-intensity interval exercise $\left(80 \% \mathrm{VO}_{2 \max }\right)$ and low-intensity continuous exercise $\left(60 \% \quad V_{2 \max }\right)$. However, it is noteworthy that in both studies blood glucose was lower during the higher intense exercise $(11,21)$. On the other hand, Boulé et al. (22) conducted a meta-analysis of 7 randomized clinical trials with a total sample of 266 individuals and found that intensity of exercise was strongly associated with the reduction of glycated hemoglobin ( $r=$ $-0.91, P=0.002)$, whereas volume was not $(r=-0.46$, $P=0.26)$.

Differences in experimental design (e.g., determination of LT and total diet kilocalories) is a possible explanation of the disagreement between the results of the present study and the results from Hiyane et al. (11) and Gross (21). Furthermore, the authors did not perform additional statistical analysis (e.g., effect size) that could have demonstrated the magnitude of the effect, which would better reflect the clinical significance of data (17).

As observed in Table 1, the exercise performed above the LT was of a greater total work (kilojoule) than that performed below LT, which might be pointed out as one limitation of the present study. While fixing the time of both sessions (20-min) to avoid influence of exercise time on physiological variables, the exercise sessions performed at different intensities (Watts) as related to LT (120 vs $80 \%$ $\mathrm{LT})$ resulted in different total work. On the other hand, despite being of different work, this approach resulted in elevation in markers of exercise intensity $\left(\mathrm{VO}_{2}, \mathrm{HR}\right.$, blood lactate) that was significantly higher for the $120 \%$, evidencing the effect of exercise intensity. The results shown in Figure 1 demonstrate that just 5-min of exercise performed at $120 \%$ LT was enough to reduce glycemia to values lower than that observed at same time points for $80 \%$ LT. Moreover, only $10-\mathrm{min}$ of exercise at $120 \%$ LT was necessary for glycemia to be lower than control and $80 \% \mathrm{LT}$, while in the entire 20-min at $80 \%$ LT the glycemia was never statistically lower than control. Therefore, a onetime 10 -min exercise at $120 \%$ LT (of which the amount of work was less than the 20th min exercise at $80 \% \mathrm{LT}$ ) was enough to reduce glycemia to values significantly lower than control. We assume that the main effect was due to exercise intensity, and not to the total work, because the glycemia at this point was reduced to values lower than control, and this was not observed even after the entire
20 -min of exercise at $80 \%$ LT. Thus, it is reasonable to infer that physical exercise that requires more work per time promotes greater glucose uptake for patients with controlled diabetes, and without secondary complications such as the participants from this study.

Physical exercise leads to an increased AMP/ATP ratio, which activates AMP-activating protein kinase (AMPK) (23). AMPK is a protein that regulates certain pathways within cells, and its activation by physical exercise is not affected by T2D (24). In turn, when activated, AMPK elicits GLUT-4 translocation to sarcolemma, leading to glucose uptake $(24,25)$. This way, it is possible that exercise performed at workload of $120 \%$ LT in the present study, leading to a higher recruitment of motor units and muscle mass activity, has elicited a more pronounced GLUT-4 translocation, resulting in a better blood glucose control of the participants. It has been shown in a previous study that activation of the involved signaling cascades is higher after exercise performed at $80 \% \mathrm{VO}_{2 \max }$ than that of $40 \%$ $\mathrm{VO}_{2 \max }$, even when energy expenditure was equalized (400 kcal), resulting in an increase of AMKP (2.8-fold) and CaMKII phosphorylation (84\%) immediately after the exercise of higher intensity (26).

Post-exercise glucose uptake can take place by insulin pathways due to effect of exercise increasing postexercise insulin sensitivity (27). However, the effects of physical exercise on insulin seem to be dependent on exercise variables (e.g., intensity and duration) and subsequent diet (19). In the present study, serum insulin decreased after exercise, which implies that glucose uptake after exercise may have occurred due to both an increased post-exercise insulin sensitivity as well as by insulinindependent pathways.

Although not measured in the present investigation, studies have suggested that IL- 6 plays a role in the glucose uptake process $(28,29)$ and that its release is increased during exercise (30). Helge et al. (31) submitted 7 healthy men to three rectangular protocols of the knee extension $(25,65$, and $85 \%$ of the maximum working capacity) and demonstrated that the higher the exercise intensity, the greater the release of IL-6 and, in turn, increased glucose uptake.

Bradykinin is a vasoactive peptide present in vascular smooth cells, endothelial cells, and in skeletal and cardiac muscles (32-34). Recently, kinin receptor (B1 and B2) knockout mice showed age-related hyperglycemia, increases of hepatic gluconeogenesis, intolerance of glucose, decreases in insulin sensibility, and functional impairment of pancreatic islets $(35,36)$. This suggests a possible role of the kalikrein-kinin system in glucose uptake and homeostasis $(35,36)$. In turn, Taguchi et al. (7) observed a blood glucose decrease in parallel with an increase in plasma bradykinin after exercise in a diabetic animal model. Moreover, researchers observed attenuation of both GLUT-4 translocation to the plasma membrane, IRS-1 phosphorylation, and PI3-K activity in skeletal muscle 
after infusion of a bradykinin antagonist-receptor (HOE140) compared to a control group. This suggests a possible role of kalikrein-kinin system, mainly bradykinin, on glucose uptake by skeletal muscles (37).

In the present study, bradykinin increased only $45 \mathrm{~min}$ after exercise performed below LT, and differences were not observed between exercise sessions. In addition, the total AUC was not affected by exercise intensities, and was unrelated to the decrease of blood glucose. The effect of exercise intensity on bradykinin pathway was not evidenced in other studies in humans as well. Simões et al. (38) did not observe an increase in plasma bradykinin, kalikrein activity, or DesArg ${ }^{9}$-bradykinin after exercise performed at $90 \%$ of LT in T2D patients. These results corroborate trials developed in animal models $(33,39)$. Schweitzer and Cartee (40) demonstrated that normal rats deficient in kininogen did not present differences in glucose transport after exercise. Other experiments developed by the same group did not show differences in blood glucose and insulin, nor in muscle glucose uptake, after exercise on B2 receptor knockout mice versus normal mice (39). Thus, it is reasonable to infer that bradykinin may not be essential for blood glucose uptake during and after exercise in T2D patients.

Another possible signaling pathway for glucose uptake, which could be a target of future investigations related to exercise intensity, is the AMPK activation from reductions in adenosine monophosphate/triphosphate and creatine/creatine phosphate ratios, or from the increase of the calcium/calmodulin complexes in skeletal muscle cells leading to signaling cascades of calcium/calmodulin protein kinases, or yet from activation of the $\mathrm{mTOR} / \mathrm{p} 70^{\mathrm{s} 6 \mathrm{~K}}$ pathway, increasing GLUT-4 translocation to the surface of membrane, promoting glucose uptake (6). The most

\section{References}

1. Haas L, Maryniuk M, Beck J, Cox CE, Duker P, Edwards L, et al. National standards for diabetes self-management education and support. Diabetes Care 2014;37 (Suppl 1): S144-S153, doi: 10.2337/dc09-S087.

2. Colberg SR, Sigal RJ, Yardley JE, Riddell MC, Dunstan DW, Dempsey PC, et al. Physical activity/exercise and diabetes: a position statement of the American Diabetes Association. Diabetes Care 2016; 39: 2065-2079, doi: 10.2337/dc161728.

3. Boulé NG, Haddad E, Kenny GP, Wells GA, Sigal RJ. Effects of exercise on glycemic control and body mass in type 2 diabetes mellitus: a meta-analysis of controlled clinical trials. JAMA 2001; 286: 1218-1227, doi: 10.1001/ jama.286.10.1218.

4. Liubaoerijijin Y, Terada T, Fletcher K, Boulé NG. Effect of aerobic exercise intensity on glycemic control in type 2 diabetes: a meta-analysis of head-to-head randomized trials. Acta Diabetol 2016; 53: 769-781, doi: 10.1007/s00592-0160870-0. important limitation of this study is the lack of a control group without T2D, which could help explain the action of bradykinin on diabetic versus healthy individuals. The findings of this study may be important for exercise physiologists, health professionals involved in the treatment of T2D, scientists whose research focuses on blood glucose response in exercise training, and individuals with $T 2 D$ who are interested in aerobic training.

In conclusion, aerobic exercise can decrease blood glucose during and after exercise performed above and below LT. However, exercise above LT demonstrated a greater blood glucose decrease than exercise performed below LT, and of a higher clinical relevance as evidenced by the effect size obtained post-exercise. The glycemia decrease occurred in parallel with an insulin decrease or stable insulin, suggesting possible involvement of insulinindependent pathways for glucose uptake during and after exercise. Bradykinin has been related to glucose homeostasis and glucose uptake, but in the present study, an increase of this peptide was observed only after $45 \mathrm{~min}$ of exercise performed below LT and was not related to the change in glucose. Thus, these results demonstrate that this peptide may not be the main factor that influences glucose uptake for T2D during and after aerobic exercise.

\section{Acknowledgments}

The authors are grateful for the students' scholarships at undergraduate (CNPq), master's (CAPES), and PhD (CAPES and CNPq) levels and for the Research Productivity Scholarships (CNPq) and materials for this research (CAPES/PROCAD No. 23038.000658/2010-70) and CNPq (No. 473598/2010-8).
5. Sigal RJ, Kenny GP, Boulé NG, Wells GA, Prud'homme D, Fortier $\mathrm{M}$, et al. Effects of aerobic training, resistance training, or both on glycemic control in type 2 diabetes: a randomized trial. Ann Intern Med 2007; 147: 357-369, doi: 10.7326/0003-4819-147-6-200709180-00005.

6. Röhling $M$, Herder $C$, Stemper T, Müssig K. Influence of acute and chronic exercise on glucose uptake. J Diabetes Rs. 2016; 2016: 1-33, doi: 10.1155/2016/2868652.

7. Taguchi T, Kishikawa H, Motoshima H, Sakai K, Nishiyama $\mathrm{T}$, Yoshizato $\mathrm{K}$, et al. Involvement of bradykinin in acute exercise-induced increase of glucose uptake and GLUT-4 translocation in skeletal muscle: studies in normal and diabetic humans and rats. Metabolism 2000; 49: 920-930, doi: 10.1053/meta.2000.6755.

8. Simões HG, Hiyane WC, Benford RE, Madrid B, Prada FA, Moreira SR, et al. Lactate threshold prediction by blood glucose and rating of perceived exertion in people with type 2 diabetes. Percept Mot Skills 2010; 111: 365-378, doi: 10.2466/06.13.15.27.PMS.111.5.365-378. 
9. Simões HG, Moreira SR, Moffatt RJ, Campbell CSG. [Methods to identify the anaerobic threshold for type-2 diabetic and non-diabetic subjects]. Arq Bras Cardiol 2010; 94: 71-78, doi: 10.1590/S0066-782X2010000100012.

10. Asano RY, Browne RAV, Sotero R da C, Sales MM, Moraes JFVN de, Campbell CSG, et al. Cycling above rather than below lactate threshold is more effective for nitric oxide release and post-exercise blood pressure reduction in individuals with type-2 diabetes. Motriz J Phys Educ UNESP 2013; 19: 633-640, doi: 10.1590/S1980-65742013000300015.

11. Hiyane WC, Sousa MV de, Moreira SR, Valle G Do, Oliveira $\mathrm{RJ}$ de, Arsa G, et al. Blood glucose responses of type-2 diabetics during and after exercise performed at intensities above and below anaerobic threshold. Rev Bras Cineantropom Desempenho Hum 2008; 10: 8, doi: 10.5007/19800037.2008v10n1p8.

12. American Diabetes Association. 2. Classification and Diagnosis of Diabetes. Diabetes Care 2016; 39 (Supplement 1): S13-S22, doi: 10.2337/dc16-S005.

13. Curtis BM, O'Keefe $\mathrm{JH}$. Autonomic tone as a cardiovascular risk factor: the dangers of chronic fight or flight. Mayo Clin Proc 2002; 77: 45-54, doi: 10.4065/77.1.45.

14. American College of Sports Medicine. ACSM's guidelines for exercise testing and prescription. Philadelphia: Lippincott Williams \& Wilkins; 2006.

15. Simões HG. Respostas hormonais e metabólicas durante os testes de determinação do limiar anaeróbio individual e lactato mínimo. Universidade Federal de São Carlos; 2002.

16. Simões HG, Campbell CSG, Kushnick MR, Nakamura A, Katsanos CS, Baldissera $\mathrm{V}$, et al. Blood glucose threshold and the metabolic responses to incremental exercise tests with and without prior lactic acidosis induction. Eur J Appl Physiol 2003; 89: 603-611, doi: 10.1007/s00421-003-0851-1.

17. Hopkins WG, Marshall SW, Batterham AM, Hanin J. Progressive statistics for studies in sports medicine and exercise science. Med Sci Sports Exerc 2009; 41: 3-13, doi: 10.1249/ MSS.0b013e31818cb278.

18. Hopkins W. A scale of magnitudes for effect statistics. A new view of statistics [Internet]. 2002. Available from: newstats. org/effectmag.html

19. Colberg SR, Sigal RJ, Fernhall B, Regensteiner JG, Blissmer BJ, Rubin RR, et al. Exercise and type 2 diabetes: the American College of Sports Medicine and the American Diabetes Association: joint position statement executive summary. Diabetes Care 2010; 33: 2692-2696, doi: 10.2337/ dc10-1548.

20. Arsa G, Lima LC de J, Motta-Santos D, Cambri LT, Campbell CSG, Lewis JE, et al. Effects of prior exercise on glycemic responses following carbohydrate ingestion in individuals with type 2 diabetes. J Clin Transl Res 2015; 1: 22-30.

21. Gross K. The acute effect of high-intensity interval training versus moderate-intensity continuous training on postprandial blood glucose regulation. Plymouth Student Sci 2015; 8: 29-47.

22. Boulé NG, Kenny GP, Haddad E, Wells GA, Sigal RJ. Metaanalysis of the effect of structured exercise training on cardiorespiratory fitness in Type 2 diabetes mellitus. Diabetologia 2003; 46: 1071-1081, doi: 10.1007/s00125-003-1160-2.

23. Carling D. The AMP-activated protein kinase cascade-a unifying system for energy control. Trends Biochem Sci 2004; 29: 18-24, doi: 10.1016/j.tibs.2003.11.005.
24. Musi N, Fujii N, Hirshman MF, Ekberg I, Fröberg S, Ljungqvist $\mathrm{O}$, et al. AMP-activated protein kinase (AMPK) is activated in muscle of subjects with type 2 diabetes during exercise. Diabetes 2001; 50: 921-927, doi: 10.2337/diabetes. 50.5.921.

25. Kurth-Kraczek EJ, Hirshman MF, Goodyear LJ, Winder WW. $5^{\prime}$ AMP-activated protein kinase activation causes GLUT4 translocation in skeletal muscle. Diabetes 1999; 48: 16671671, doi: 10.2337/diabetes.48.8.1667.

26. Egan B, Carson BP, Garcia-Roves PM, Chibalin AV, Sarsfield FM, Barron N, et al. Exercise intensity-dependent regulation of peroxisome proliferator-activated receptor $\gamma$ coactivator- $1 \alpha$ mRNA abundance is associated with differential activation of upstream signalling kinases in human skeletal muscle. J Physiol 2010; 588: 1779-1790, doi: 10.1113/ jphysiol.2010.188011.

27. Hoy AJ, Turner N. New insight into the mechanism by which acute physical exercise ameliorates insulin resistance. J Physiol 2008; 586: 2251-2252, doi: 10.1113/jphysiol.2008. 152991.

28. Stouthard JM, Oude Elferink RP, Sauerwein HP. Interleukin6 enhances glucose transport in 3T3-L1 adipocytes. Biochem Biophys Res Commun 1996; 220: 241-245, doi: 10.1006/bbrc.1996.0389.

29. Tsigos C, Papanicolaou DA, Kyrou I, Defensor R, Mitsiadis CS, Chrousos GP. Dose-dependent effects of recombinant human interleukin-6 on glucose regulation. J Clin Endocrinol Metab 1997; 82: 4167-4170, doi: 10.1210/jcem.82.12.4422.

30. Steensberg A, van Hall G, Osada T, Sacchetti M, Saltin B, Klarlund Pedersen B. Production of interleukin- 6 in contracting human skeletal muscles can account for the exercise-induced increase in plasma interleukin-6. J Physiol 2000; 529 (Part 1): 237-242, doi: 10.1111/j.1469-7793.2000. 00237.x.

31. Helge JW, Stallknecht B, Pedersen BK, Galbo H, Kiens B, Richter EA. The effect of graded exercise on IL-6 release and glucose uptake in human skeletal muscle. $J$ Physiol 2003; 546 (Part 1): 299-305, doi: 10.1113/jphysiol.2002. 030437.

32. Jaffa AA, Durazo-Arvizu R, Zheng D, Lackland DT, Srikanth $\mathrm{S}$, Garvey WT, et al. Plasma prekallikrein: a risk marker for hypertension and nephropathy in type 1 diabetes. Diabetes 2003; 52: 1215-1221, doi: 10.2337/diabetes.52.5.1215.

33. Bhoola KD, Figueroa CD, Worthy K. Bioregulation of kinins: kallikreins, kininogens, and kininases. Pharmacol Rev 1992; 44: 1-80.

34. Oza NB, Schwartz JH, Goud HD, Levinsky NG. Rat aortic smooth muscle cells in culture express kallikrein, kininogen, and bradykininase activity. J Clin Invest 1990; 85: 597-600, doi: $10.1172 / \mathrm{JCl} 114479$.

35. Barros CC, Haro A, Russo FJVP, Schadock I, Almeida SS, Ribeiro RA, et al. Altered glucose homeostasis and hepatic function in obese mice deficient for both kinin receptor genes. PLoS One 2012; 7: e40573, doi: 10.1371/journal. pone. 0040573 .

36. Barros CC, Haro A, Russo FJ, Schadock I, Almeida SS, Reis FC, et al. Bradykinin inhibits hepatic gluconeogenesis in obese mice. Lab Invest 2012; 92: 1419-1427, doi: 10.1038/ labinvest.2012.105.

37. Pauli JR, Cintra DE, Souza CT de, Ropelle ER. [New mechanisms by which physical exercise improves insulin 
resistance in the skeletal muscle]. Arq Bras Endocrinol Metabol 2009; 53: 399-408, doi: 10.1590/S0004-27302009 000400003.

38. Simões HG, Asano RY, Sales MM, Browne RAV, Arsa G, Motta-Santos D, et al. Type 2 diabetes elicits lower nitric oxide, bradykinin concentration and kallikrein activity together with higher DesArg(9)-BK and reduced post-exercise hypotension compared to non-diabetic condition. PLoS One 2013; 8: e80348, doi: 10.1371/journal.pone.0065804.
39. Schweitzer GG, Castorena CM, Hamada T, Funai K, Arias EB, Cartee GD. The B2 receptor of bradykinin is not essential for the post-exercise increase in glucose uptake by insulin-stimulated mouse skeletal muscle. Physiol Res 2011; 60: 511-519.

40. Schweitzer GG, Cartee GD. Post-exercise skeletal muscle glucose transport is normal in kininogen-deficient rats. Med Sci Sports Exerc 2011; 43: 1148-1153, doi: 10.1249/MSS. Ob013e31820a7f65. 